\title{
Self-assembly of nanoscale lateral segregation profiles
}

\author{
R. Stania,,${ }^{1,2,{ }^{*}}$ W. Heckel, ${ }^{3}$ I. Kalichava, ${ }^{2}$ C. Bernard, ${ }^{1}$ T. C. Kerscher, ${ }^{3}$ H. Y. Cun, ${ }^{1}$ P. R. Willmott, ${ }^{2}$ B. Schönfeld, ${ }^{4}$ \\ J. Osterwalder, ${ }^{1}$ S. Müller, ${ }^{3}$ and T. Greber ${ }^{1}$ \\ ${ }^{1}$ Physik-Institut, Universität Zürich, Winterthurerstrasse 190, 8057 Zürich, Switzerland \\ ${ }^{2}$ Paul Scherrer Institut, 5232 Villigen PSI, Switzerland \\ ${ }^{3}$ Hamburg University of Technology, Denickestraße 15, 21073 Hamburg, Germany \\ ${ }^{4}$ Laboratory of Metal Physics and Technology, Department of Materials, ETH Zürich, 8093 Zürich, Switzerland
}

(Received 27 November 2015; revised manuscript received 26 January 2016; published 7 April 2016)

\begin{abstract}
The surface segregation profile of an intermetallic compound becomes vertically and laterally modulated upon epitaxial growth of a single-layer hexagonal boron nitride $(h$-BN) nanomesh. $h$-BN on PtRh(111) forms an 11-on-10 superhoneycomb, such as that on $\mathrm{Rh}(111)$ [Corso et al., Science 303, 217 (2004)], though with a smaller lattice constant of $2.73 \mathrm{~nm}$. X-ray photoelectron diffraction shows that the $h$-BN layer reduces the $\mathrm{Pt}$ enrichment of the first layer by promoting site swapping of about $10 \mathrm{Pt}-\mathrm{Rh}$ pairs within the $10 \times 10$ unit cell between the first and second layers. This segregation profile is confirmed by density-functional-theory-based cluster-expansion calculations. Generally, a strong modulation of the $h$-BN bonding strength and a higher affinity to one of the constituents leads to self-assembly of top layer patches underneath the nanomesh pores.
\end{abstract}

DOI: 10.1103/PhysRevB.93.161402

Surface segregation is an important process in intermetallic compounds [1]. The surface enrichment of a certain atomic species has many practical applications, as in hydrogen storage [2] or nanocatalysis [3]. The deviation from the bulk composition is driven by strain release and different surface energies of the constituent elements [4]. Today's computational power may tackle the problem of surface segregation and ordering of alloy surfaces in the presence of adsorbates by a concerted effort of $a b$ initio density functional theory, cluster expansion, and Monte Carlo simulations [5]. For PtRh, comparisons to experiments [6] show excellent agreement [7] and phenomena like carbon-induced segregation can be understood [8].

Lateral segregation on the micrometer scale was observed in heterogeneous catalysis in artificial heterostructures [9]. Here we report on lateral segregation profiles in the top layers of $\operatorname{PtRh}(111)$ on the nanometer scale. They are created during the growth of a single layer of hexagonal boron nitride ( $h$-BN), which imposes the formation of a superhoneycomb "nanomesh." We show that the site- and species-selective interaction of the overlayer strongly affects the segregation profiles, both vertically and laterally.

$h$-BN grown on $\mathrm{Rh}(111)$ forms a corrugated monolayer with a 13-on-12 superhoneycomb structure known as the $h$-BN nanomesh $[10,11]$. In contrast, $h$-BN on $\mathrm{Pt}(111)$ forms a flat layer with an approximate 10-on-9 superstructure, due to weaker and less selective bonding than in the $h$ $\mathrm{BN} / \mathrm{Rh}(111)$ nanomesh $[12,13]$. The growth of $h-\mathrm{BN}$ on $\operatorname{PtRh}(111)$ produces as well a nanomeshlike superstructure. Segregation distinct from the bare surface occurs in the topmost layers of the substrate during growth of $h$-BN, and $\mathrm{Rh}$ atoms form patches under the pores of the nanomesh, which are surrounded by $\mathrm{Pt}$ atoms underneath the wires. This overlayer-assisted segregation signifies a new tool for self-assembly in intermetallic compounds and has potential impact in intercalation chemistry [14-16], the production of

*Corresponding author: rostania@physik.uzh.ch nanometer-sized holes in $h$-BN [17], or in the formation of new magnetic structures, to name three potential applications. Experimental and calculating details are described in the Supplemental Material [18].

Figure 1 shows X-ray photoemission spectra (XPS) of the $\mathrm{Rh} 3 d$ and the $\mathrm{Pt} 4 d_{5 / 2}$ peaks from annealed $\mathrm{PtRh}$ and singlelayer $h$-BN on PtRh(111). The platinum surface segregation can be directly seen by comparing normal and grazing emission spectra for both systems: The Pt:Rh ratio increases in going from normal $\left(\theta=0^{\circ}\right)$ to grazing $\left(\theta=78^{\circ}\right)$ emission. For grazing emission we chose an azimuthal emission angle away from any forward-scattering peaks. From the mean free path in PtRh at the given electron kinetic energy, the probing depths are 1.25 and $0.26 \mathrm{~nm}$, respectively. The $h$-BN layer attenuates the photoelectron emission; however, this does not affect the observed $\mathrm{Pt}: \mathrm{Rh}$ ratio, because the relative kinetic energy difference between the peaks in Fig. 1 is less than $1 \%$.

From a comparison of the grazing emission spectra of bare and $h$-BN covered PtRh [Figs. 1(b) and 1(d)] it is seen that in the latter case the $\mathrm{Pt}: \mathrm{Rh}$ ratio decreases, which indicates, given the small probing depth, Rh segregation to the top layer. If the peak areas are evaluated and normalized with the corresponding photoemission cross sections, the atomic concentrations for the given probing depth can be determined [18]. The four pie diagrams in Fig. 1 show that all spectra indicate excess of Pt with respect to the bulk stoichiometry. The Pt content is largest for the grazing spectrum of bare PtRh [Fig. 1(b)].

For a complete picture of the influence of $h$-BN on segregation, as suggested by the XPS data in Fig. 1, the structure has been studied with four more surface-science techniques. It was thereby found that the $h$-BN forms a commensurate superhoneycomb (nanomesh) structure, similar to the case of $\operatorname{Rh}(111)$ [11], but not to that of $\operatorname{Pt}(111)[12,37,38]$. In addition, it induces Rh enrichment in the top layer. Figure 2(a) displays low-energy electron-diffraction data of $h$-BN/PtRh, where a perfect, highly ordered superstructure that is aligned to the substrate is observed. The superstructure dimensions are quantified using surface x-ray diffraction [39]. First, the unit cell size of the bimetallic bulk crystal was determined to be 

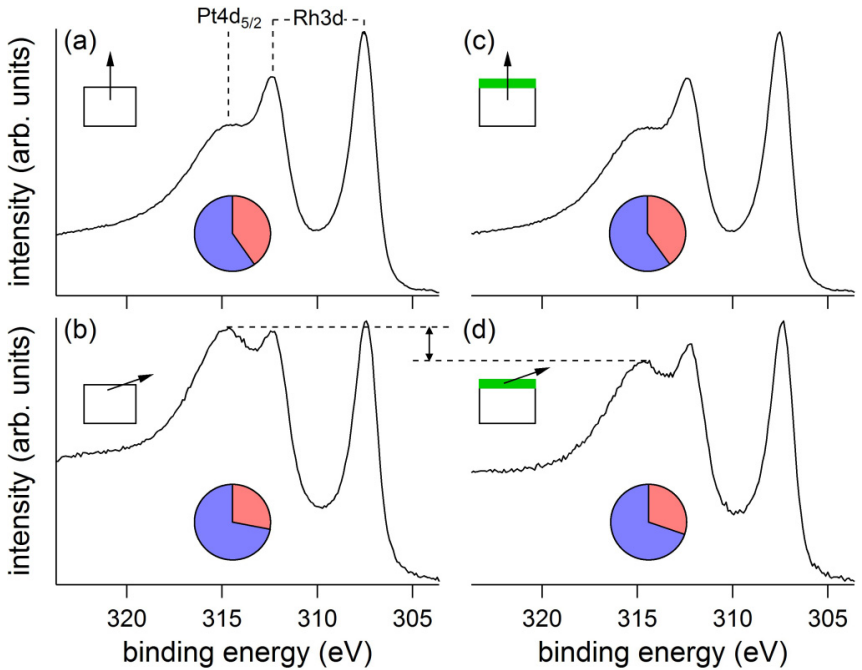

FIG. 1. $\mathrm{Mg} \mathrm{K} \alpha$ excited $\mathrm{Rh} 3 d$ and $\mathrm{Pt} 4 d_{5 / 2}$ x-ray photoelectron spectra of bare PtRh at (a) normal, (b) grazing and $h-\mathrm{BN} / \mathrm{PtRh}$ at (c) normal and (d) grazing emission angles. The spectra are normalized to the $\mathrm{Rh} 3 d_{5 / 2}$ intensity. The relative increase of the $\mathrm{Pt} 4 d_{5 / 2}$ signal at $315 \mathrm{eV}$ in going from normal to grazing emission indicates $\mathrm{Pt}$ surface segregation. The decrease of the grazing Pt signal for $h$ $\mathrm{BN} / \mathrm{PtRh}$ indicates $h$-BN-induced $\mathrm{Rh}$ segregation to the top layer. The pie diagrams represent the $\mathrm{Pt}$ (blue) and the $\mathrm{Rh}$ (red) atomic concentrations for the respective probing depths.

within $0.1 \%$ of that expected from Vegard's law for a 50:50 atomic mixture. Figure 2(b) shows an in-plane diffraction scan in units of the PtRh surface lattice $(h, k, l)$, at $l=1.2$ and $k=0$, and along $h$. In addition to the bulk crystal truncation rod at $h=$ 1 , the superstructure signal is seen at $h=9 / 10$ and $11 / 10$. The superstructure unit cell thus consists of $(11 \times 11) h$-BN units on top of $(10 \times 10)$ PtRh units, with a superlattice constant of $2.729 \pm 0.001 \mathrm{~nm}$. The $\frac{11}{10}$ rod mainly reflects scattering off the BN lattice and would also be expected for noninteracting $h$-BN. The presence of the $\frac{9}{10}$ rod, however, proves commensurability and a periodic strain between PtRh and $h$-BN [40]. Therefore, the lattice mismatch between the two $(1 \times 1)$ unit cells of $-8.4 \%$ is relaxed by the superstructure to $+0.8 \%$, which indicates that the $h$-BN in the $10 \times 10$ structure undergoes weak compressive strain, as is the case for $h$-BN/Rh(111).

The electronic structure of $h$-BN/PtRh(111) shows a $\sigma$ band splitting, as also observed for the $h$-BN/Rh(111) nanomesh [10,11]. In Fig. 2(c) normal emission He I $\alpha$ spectra for bare $\operatorname{PtRh}(111)$ and $h$-BN/PtRh(111) are shown. For $h$ - $\mathrm{BN} / \mathrm{PtRh}(111)$ the spectrum is dominated by the $\sigma$ band that appears split by $0.9 \mathrm{eV}$ into $\sigma_{\alpha}$ and $\sigma_{\beta}$ components. The $\sigma$ band features coincide with the PtRh band structure related feature at $3.9 \mathrm{eV}$ binding energy that has, however, a distinct dispersion [18]. The $\sigma$ band splitting is a strong indication of the formation of a nanomesh structure with two distinct electronic regions defined by the wires and pores [11]. A further influence of the $h$-BN overlayer on the surface electronic structure is observed in the photoemission intensity at the Fermi level. The spectral density increases upon $h$-BN layer growth. Since $h$-BN has no states at the Fermi level, this must be due to changes in the top layer of the PtRh. (a)

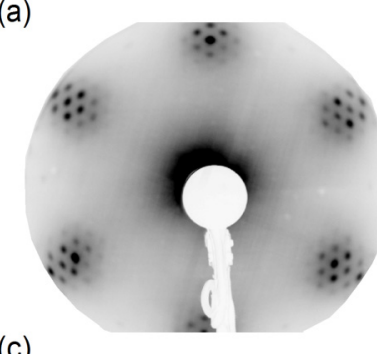

(c)
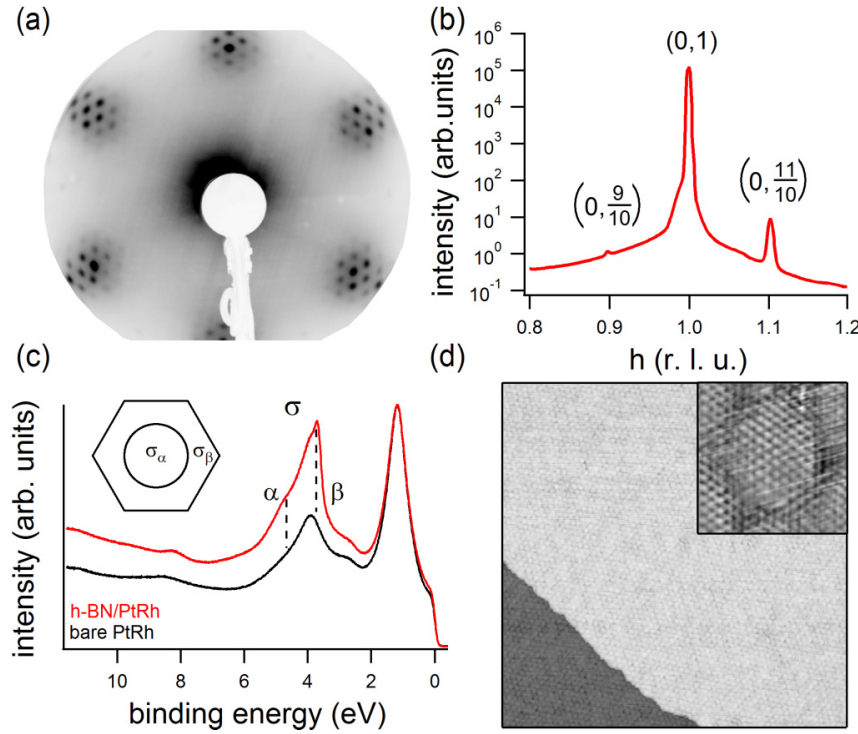

FIG. 2. Identification of the nanomesh superstructure of $h$ $\mathrm{BN} / \mathrm{PtRh}(111)$. (a) Low-energy electron diffraction (LEED) pattern $(E=70 \mathrm{eV})$. The $1 \times 1 \mathrm{PtRh}(111)$ principal spots are surrounded by $h$-BN-induced superstructure spots. (b) Surface x-ray diffraction (SXRD) along the $h$ direction showing the crystal truncation rod and $h$-BN-derived $11 / 10$ and $9 / 10$ superstructure rods. (c) Normal emission angular resolved $\mathrm{He} \mathrm{I} \alpha$ spectra for bare PtRh (black) and $h$-BN/PtRh(111) (red). The spectra are normalized to the intensity of the valence band peak at $\sim 1 \mathrm{eV}$. The dashed lines indicate the positions of the $\sigma$ bands of the $h$-BN layer corresponding to the pores $(\alpha)$ or wires $(\beta)$ as shown in the inset. (d) Scanning tunneling microscopy images taken in constant current mode $\left(150 \times 150 \mathrm{~nm}^{2}\right.$, $I_{t}=1 \mathrm{nA}, U_{t}=5 \mathrm{mV}$, drift corrected $)$. The inset $\left(3.7 \times 3.7 \mathrm{~nm}^{2}, I_{t}=\right.$ $1.5 \mathrm{nA}, U_{t}=0.5 \mathrm{mV}$ ) reveals, after subtraction of the corrugation, the superhoneycomb unit cell with atomic resolution.

The structure of the unit cell was further investigated with scanning tunneling microscopy. Figure 2(d) shows large terraces of an ordered hexagonal structure similar to $h$ $\mathrm{BN} / \mathrm{Rh}(111)[10,11]$. The inset shows atomic resolution after subtraction of the superhoneycomb corrugation and exhibits the $11 \times 11$ superstructure of the $h$-BN. In contrast to the $h$ $\mathrm{BN} / \mathrm{Rh}(111)$ nanomesh where the superhoneycomb is imaged with pores surrounded by wires, here the contrast of the image is inverted. This is not found to systematically depend on the tunneling voltage, although certain tip conditions produced images with reversed corrugation. We propose that the imaged corrugation in $h$-BN/PtRh(111) has its origin in the higher local density of states of $\mathrm{Rh}$ as compared to $\mathrm{Pt}$ [1].

To quantify the Rh enrichment in the top layer we applied angle scanned $\mathrm{x}$-ray photoelectron diffraction (XPD) [41]. Figures 3(a)-3(d) show XPD patterns of the Pt $4 f_{7 / 2}\left(E_{\text {kin }}=\right.$ $1175.5 \mathrm{eV})$ and the Rh $3 d_{5 / 2}\left(E_{\mathrm{kin}}=939.8 \mathrm{eV}\right)$ XPS lines of bare PtRh and $h$-BN/PtRh. The stereographic maps display patterns as observed for fcc(111) structures [42]. Thus, Rh and Pt atoms share the same lattice sites and any preferential ordering is not obvious. To visualize the changes in the substrate upon growth of $h$-BN the cross ratio $X$ of the four XPD maps is displayed in Fig. 3(e) with

$$
X(\theta, \phi)=\frac{I_{\mathrm{Rh}(h-\mathrm{BN})}}{I_{\mathrm{Pt}(h-\mathrm{BN})}} \cdot \frac{I_{\mathrm{Pt}(\text { bare })}}{I_{\mathrm{Rh}(\text { bare })}},
$$




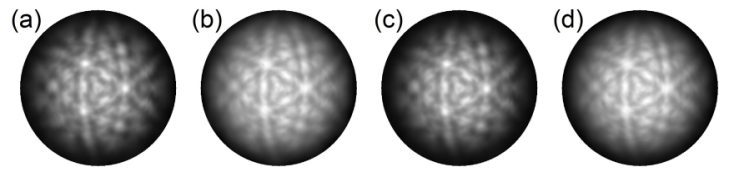

(e)
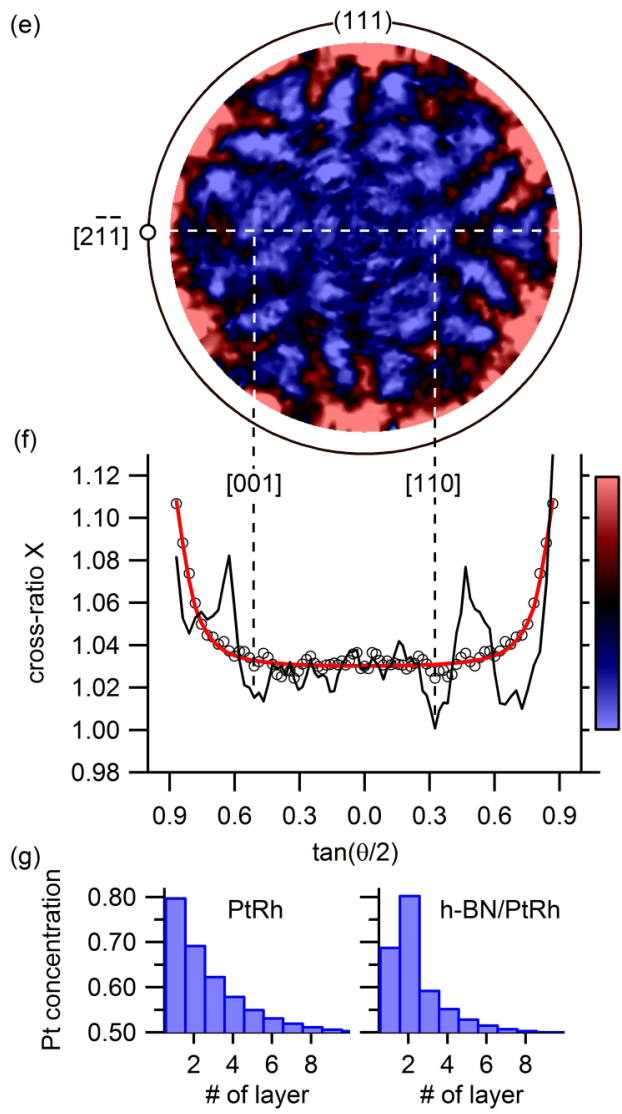

FIG. 3. $\mathrm{Mg} \mathrm{K} \alpha$ excited x-ray photoelectron diffraction (XPD) patterns for $\mathrm{Rh} 3 d_{5 / 2}\left(E_{\mathrm{kin}}=939.8 \mathrm{eV}\right)$ and $\mathrm{Pt} 4 f_{7 / 2}\left(\mathrm{E}_{k i n}=\right.$ $1175.5 \mathrm{eV}$ ) emission. (a) Rh bare, (b) Pt bare, (c) $\mathrm{Rh} h-\mathrm{BN}$, and (d) Pt $h$-BN. (e) Cross-ratio $X$ [Eq. (1)]. (f) Polar dependence of $X$ on the azimuths containing $\langle 110\rangle$ and $\langle 001\rangle$ (solid line) and the azimuthal average (open circles). The red line is $X$ as obtained from the Pt profiles in $(\mathrm{g})$. (g) Pt concentrations as a function of the layer number. Note the swap of about $10 \%$ between the first and second layers.

where $I$ is the intensity for given polar and azimuthal emission angles $\theta$ and $\phi$. The cross ratio eliminates systematic errors due to the angular transmission or photoemission cross sections and allows very accurate quantitative statements on changes in the structure. High (low) $X$ values indicate an increased relative $\mathrm{Rh}(\mathrm{Pt})$ contribution to the XPD patterns after growth of $h$-BN, and are shown in red (blue). The Rh contribution increases toward high polar angles, consistent with the XPS data in Fig. 1. Furthermore, $X$ displays anisotropy in the azimuthal angles. The patterns resemble those of emitters in the second layer, where $\langle 110\rangle$ and $\langle 001\rangle$ forward-scattering directions are most prominent [18]. Figure 3(e) thus indicates that upon nanomesh formation, Rh atoms in the second layer swap sites with $\mathrm{Pt}$ atoms in the top layer, where no forward scattering is expected for polar emission angles smaller than $80^{\circ}$.

Figure 3(f) shows the polar cut of $X$ containing the $\langle 110\rangle$ and $\langle 001\rangle$ directions, and the $\phi$-averaged values for a given polar angle (black circles). These average values may be fit to cross ratios from a model of the $\mathrm{Pt}$ and $\mathrm{Rh}$ concentration in each layer, where the mean free path of the Pt $4 f(1.47 \mathrm{~nm})$ and the $\mathrm{Rh} 3 d(1.25 \mathrm{~nm})$ electrons enter, though the cross sections and the instrument transmission cancel in $X$. In the model, we adopt an exponential decrease of the excess Pt to the bulk value, and for the $h$-BN covered case, we allow a distribution deviating from the exponential decrease in the first two layers. The red line in Fig. 3(f) is the result of the two Pt concentration profiles in Fig. 3(g). We see that $80 \%$ of the top layer of bare $\mathrm{PtRh}$ consists of $\mathrm{Pt}$ atoms compared to $69 \%$ of $\mathrm{Pt}$ atoms in the second layer. After growth of $h$-BN the fit indicates $69 \% \mathrm{Pt}$ in the top layer and $81 \% \mathrm{Pt}$ in the second layer, which confirms the swapping of $\mathrm{Pt}$ and $\mathrm{Rh}$ in the first two layers and quantifies the amount to about 10 swaps per $10 \times 10$ unit cell. The fit results in a slightly larger (1.8 and 2.3 layers) decay constant of the excess Pt for the bare surface, which indicates that the $h$-BN influences the segregation profile beneath the second layer.

The swap of Rh and Pt atoms in the first two layers must be driven by an energy gain due to a stronger bonding of $h$-BN to $\mathrm{Rh}$ than to Pt [43]. Given the experimental fact that PtRh adopts a nanomesh structure, we propose a model where the

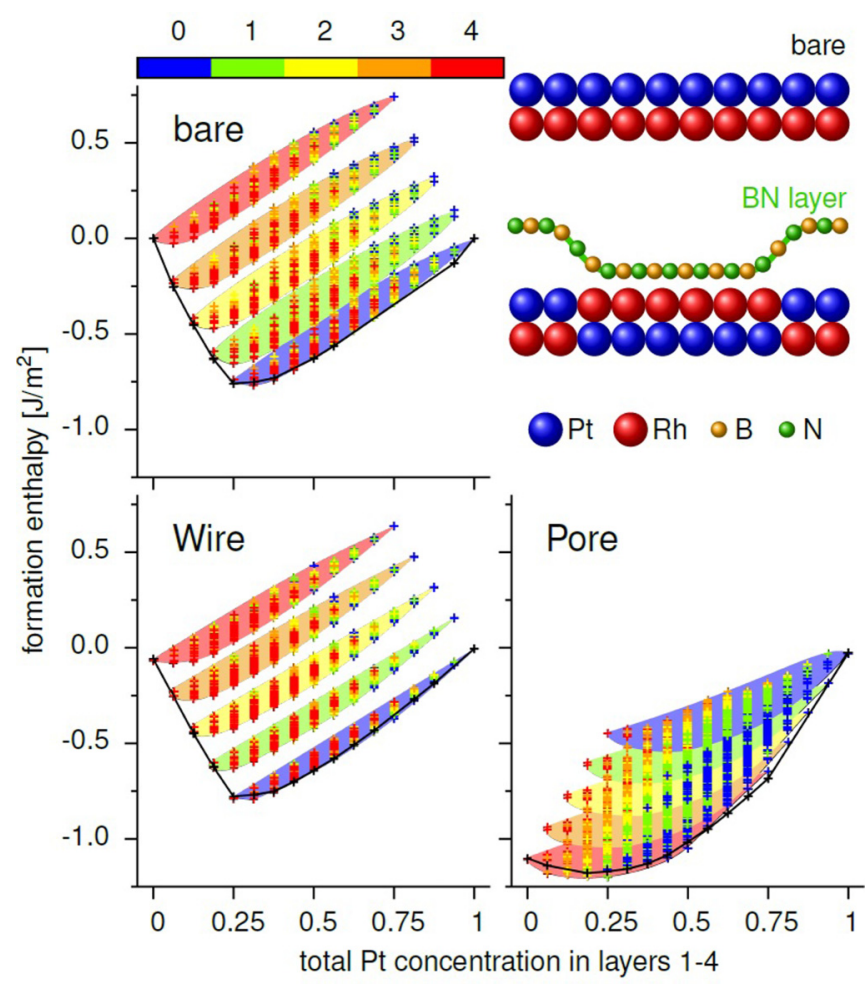

FIG. 4. Cluster-expansion energies (+) of approximately 13000 different substrate structures each for the bare PtRh(111) surface (top left), for one of the $h$-BN wire regions (bottom left), and for the $h$-BN pore region (bottom right) as a function of $\mathrm{Pt}$ concentration. Information about the segregation profile in the two topmost substrate layers is color-coded by the number of Pt atoms per layer. The filled areas code for the topmost layer, and the colored + for the second layer (blue denoting an all-Pt, red an all-Rh layer). The energy scale in all plots is given with respect to the bare case. The top right panel summarizes the lateral segregation scenario where the first two PtRh layers in the unit cell are represented by chains of 10 atoms. 
number of rhodium atoms accumulates in the pore regions of the first layer, and that this effect is fairly pronounced.

This lateral segregation picture is corroborated with an $a b$ initio study that combines density functional theory (DFT) calculations with a cluster expansion, as has also been applied for bare PtRh surfaces [7,8,18,44,45]. Our surface-slab model is a $2 \times 2 \mathrm{PtRh}(111)$ substrate cell with four substrate layers (on top of fixed bulklike PtRh layers) and one (or none) $h$-BN layer on top of the substrate [18]. The total of 16 substrate sites can be arbitrarily occupied by $\mathrm{Pt}$ or $\mathrm{Rh}$. The $h$-BN layer on top was placed in different positions in order to mimic locally the pore $(\alpha)$ and the wire $(\beta)$ regions of the superhoneycomb lattice. For each region, the energetics of more than 350 fully relaxed DFT input structures was used as $a b$ initio input for the cluster expansions, which then gave access to the energies of all possible $\mathrm{Pt}$ and $\mathrm{Rh}$ configurations in the substrate layers (roughly 13000 per region). This comprehensive scan enables us to directly see the influence of $\mathrm{BN}$ on the substrate's segregation profile at $T=0 \mathrm{~K}$.

Figure 4 shows the formation enthalpies for bare PtRh, and for $h$-BN/PtRh with wire and pore registry for all atomic configurations in the first (color-coded filled areas) and the second (color-coded crosses + ) layer. For example, the lowest energy configuration for bare and wire $h$-BN/PtRh has $0 \mathrm{Rh}$ atoms in the first and $4 \mathrm{Rh}$ atoms in the second layer, while this situation inverts for pore $h$-BN/PtRh, and lowers its energy. The energy difference between the different regions is the driving force for the laterally modulated segregation profile. The color-filled areas clearly demonstrate that the first-layer occupation dominates the energetics. For the bare case and the wire regions of $h$-BN/PtRh, Pt segregates to the topmost layer while the second layer is Pt depleted. The pore region, however, favors $\mathrm{Rh}$ segregation in the first and $\mathrm{Rh}$ depletion in the second layer. These results are sketched in the top right panel in Fig. 4, where the theoretical PtRh segregation profile with and without $h$-BN is shown for the first two layers. This is in perfect qualitative agreement with the lateral segregation model drawn from the experiments. The quantitative difference is assigned to the fact that the PtRh diffusion and thus the segregation freeze at finite temperatures, where entropy maintains $\mathrm{Rh}$ in the top layer of the bare surface.

In conclusion we present direct evidence that the top layer of PtRh is enriched with Rh, while the second layer is depleted of $\mathrm{Rh}$, when a single layer of $h-\mathrm{BN}$ is grown on top. This swap imposes a lateral surface segregation profile, which is corroborated by (i) the $h$-BN displaying a nanomeshlike structure with two distinct $\mathrm{BN}$ bonding regimes, and (ii) by the fact that $\mathrm{BN}$ binds more strongly to $\mathrm{Rh}$ than to Pt. The lateral segregation scenario, where hexagonally packed $\mathrm{Rh}$ patches with a diameter of about $2 \mathrm{~nm}$ are formed, will have an impact on confined catalysis beneath a single layer of hexagonal boron nitride, or its use as a molecular template with an electronic structure modulation that exceeds those of nanomesh templates grown on pure metals. The findings are general and we expect lateral segregation profiles on the nanometer scale also for magnetic intermetallic alloys such as FePt. If one should succeed in removing the boron nitride without affecting the PtRh structure beneath, very high reactivities of these surfaces will be at hand.

Calculations were performed on the Vienna Scientific Cluster (VSC2). The surface diffraction measurements were performed at the Materials Science beamline of the Swiss Light Source, Paul Scherrer Institut, Villigen. This work was supported by the Swiss National Science Foundation.
[1] M. Schmid and P. Varga, Chem. Phys. Solid Surf. 10, 118 (2002).

[2] H. C. Siegmann, L. Schlapbach, and C. R. Brundle, Phys. Rev. Lett. 40, 972 (1978).

[3] K. J. Mayrhofer, V. Juhart, K. Hartl, M. Hanzlik, and M. Arenz, Z. Angew. Chem., Int. Ed. 48, 3529 (2009).

[4] F. F. Abraham, Phys. Rev. Lett. 46, 546 (1981).

[5] B. C. Han, A. V. der Ven, G. Ceder, and B.-J. Hwang, Phys. Rev. B 72, 205409 (2005).

[6] E. Platzgummer, M. Sporn, R. Koller, S. Forsthuber, M. Schmid, W. Hofer, and P. Varga, Surf. Sci. 419, 236 (1999).

[7] P. Welker, O. Wieckhorst, T. C. Kerscher, and S. Müller, J. Phys.: Condens. Matter 22, 384203 (2010).

[8] T. C. Kerscher, W. Landgraf, R. Podloucky, and S. Müller, Phys. Rev. B 86, 195420 (2012).

[9] F. Esch, S. Günther, E. Schütz, A. Schaak, I. Kevrekidis, M. Marsi, M. Kiskinova, and R. Imbihl, Surf. Sci. 443, 245 (1999).

[10] M. Corso, W. Auwärter, M. Muntwiler, A. Tamai, T. Greber, and J. Osterwalder, Science 303, 217 (2004).

[11] S. Berner, M. Corso, R. Widmer, O. Groening, R. Laskowski, P. Blaha, K. Schwarz, A. Goriachko, H. Over, S. Gsell et al., Z. Angew. Chem., Int. Ed. 46, 5115 (2007).

[12] E. Ćavar, R. Westerström, A. Mikkelsen, E. Lundgren, A. Vinogradov, M. L. Ng, A. Preobrajenski, A. Zakharov, and N. Mårtensson, Surf. Sci. 602, 1722 (2008).
[13] A. Preobrajenski, M. Nesterov, M. L. Ng, A. Vinogradov, and N. Mårtensson, Chem. Phys. Lett. 446, 119 (2007).

[14] P. Sutter, J. T. Sadowski, and E. A. Sutter, J. Am. Chem. Soc. 132, 8175 (2010).

[15] E. Grånäs, M. Andersen, M. A. Arman, T. Gerber, B. Hammer, J. Schnadt, J. N. Andersen, T. Michely, and J. Knudsen, J. Phys. Chem. C 117, 16438 (2013).

[16] A. Nierhoff, C. Conradsen, D. McCarthy, T. P. Johansson, J. Knudsen, and I. Chorkendorff, Catal. Today 244, 130 (2015).

[17] H. Y. Cun, M. Iannuzzi, A. Hemmi, J. Osterwalder, and T. Greber, ACS Nano 8, 7423 (2014).

[18] See Supplemental Material at http://link.aps.org/supplemental/ 10.1103/PhysRevB.93.161402 for experimental and calculating details. References [19-36] appear therein.

[19] T. Greber, O. Raetzo, T. Kreutz, P. Schwaller, W. Deichmann, E. Wetli, and J. Osterwalder, Rev. Sci. Instrum. 68, 4549 (1997).

[20] H. Cun, M. Iannuzzi, A. Hemmi, S. Roth, J. Osterwalder, and T. Greber, Nano Lett. 13, 2098 (2013).

[21] C. Steiner, B. Schönfeld, M. J. Portmann, M. Kompatscher, G. Kostorz, A. Mazuelas, T. Metzger, J. Kohlbrecher, and B. Demé, Phys. Rev. B 71, 104204 (2005).

[22] K. D. Sattler, Handbook of Nanophysics (CRC, Boca Raton, FL, 2010).

[23] J. J. Yeh and I. Lindau, At. Data. Nucl. Data Tables 32, 1 (1985). 
[24] G. Kresse and J. Furthmüller, Phys. Rev. B 54, 11169 (1996).

[25] G. Kresse and J. Furthmüller, Comp. Mat. Sci. 6, 15 (1996).

[26] J. M. Sanchez, F. Ducastelle, and D. Gratias, Physica A 128, 334 (1984).

[27] D. Lerch, O. Wieckhorst, G. L. W. Hart, R. W. Forcade, and S. Müller, Model. Simulat. Mater. Sci. Eng. 17, 055003 (2009).

[28] P. E. Blöchl, Phys. Rev. B 50, 17953 (1994).

[29] G. Kresse and D. Joubert, Phys. Rev. B 59, 1758 (1999).

[30] J. P. Perdew, J. A. Chevary, S. H. Vosko, K. A. Jackson, M. R. Pederson, D. J. Singh, and C. Fiolhais, Phys. Rev. B 46, 6671 (1992).

[31] J. Klimeš, D. R. Bowler, and A. Michaelides, J. Phys.: Condens. Matter 22, 022201 (2010).

[32] J. Klimeš, D. R. Bowler, and A. Michaelides, Phys. Rev. B 83, 195131 (2011).

[33] H. J. Monkhorst and J. D. Pack, Phys. Rev. B 13, 5188 (1976).

[34] G. Makov and M. C. Payne, Phys. Rev. B 51, 4014 (1995).

[35] J. Neugebauer and M. Scheffler, Phys. Rev. B 46, 16067 (1992).

[36] F. J. García de Abajo, M. A. Van Hove, and C. S. Fadley, Phys. Rev. B 63, 075404 (2001).
[37] F. Müller, K. Stöwe, and H. Sachdev, Chem. Mater. 17, 3464 (2005).

[38] A. B. Preobrajenski, A. S. Vinogradov, M. L. Ng, E. avar, R. Westerström, A. Mikkelsen, E. Lundgren, and N. Mårtensson, Phys. Rev. B 75, 245412 (2007).

[39] P. Willmott, D. Meister, S. Leake, M. Lange, A. Bergamaschi, M. Böge, M. Calvi, C. Cancellieri, N. Casati, A. Cervellino et al., J. Synchr. Rad. 20, 667 (2013).

[40] D. Martoccia, P. R. Willmott, T. Brugger, M. Björck, S. Günther, C. M. Schlepütz, A. Cervellino, S. A. Pauli, B. D. Patterson, S. Marchini et al., Phys. Rev. Lett. 101, 126102 (2008).

[41] C. Fadley, Progr. Surf. Sci. 16, 275 (1984).

[42] J. Wider, F. Baumberger, M. Sambi, R. Gotter, A. Verdini, F. Bruno, D. Cvetko, A. Morgante, T. Greber, and J. Osterwalder, Phys. Rev. Lett. 86, 2337 (2001).

[43] R. Laskowski and P. Blaha, Phys. Rev. B 81, 075418 (2010).

[44] S. Müller, M. Stöhr, and O. Wieckhorst, Appl. Phys. A 82, 415 (2006).

[45] S. B. Maisel, T. C. Kerscher, and S. Müller, Acta Mater. 60, 1093 (2012). 